\title{
Bussulfano e melfalano como regime de condicionamento para o transplante autogênico de células-tronco hematopoéticas na leucemia mielóide aguda em primeira remissão completa
}

\author{
Busulfan and melphalan as conditioning regimen for autologous hematopoietic stem cell \\ transplantation in acute myeloid leukemia in first complete remission
}

\author{
Nadjanara D. Bueno \\ Frederico L. Dulley \\ Rosaura Saboya \\ José U. Amigo Filho \\ Lílian Piron-Ruiz \\ Daniel Sturaro \\ Dalton A. F. Chamone
}

\begin{abstract}
Vinte e dois pacientes consecutivos portadores de leucemia mielóide aguda (LMA) em primeira remissão completa $\left(1^{a} R C\right)$ submetidos a transplante de células-tronco hematopoéticas autogênico (TCTH Auto) condicionados com bussulfano e melfalano (Bu/Mel) foram selecionados entre 1993 e 2006. A probabilidade de sobrevida global (SG) pelo método de Kaplan-Meier foi de 57,5\% após 36 meses, com "plateau" aos 20 meses após o transplante. Fatores como sexo, classificação Franco-Americana-Britânica (FAB) da LMA, tratamento de indução, consolidação intensiva, remissão após o primeiro ciclo de indução e fonte de células não tiveram impacto na sobrevida. Pela análise citogenética, um paciente de mau prognóstico submetido ao procedimento, foi a óbito um ano após o transplante. Nove pacientes foram a óbito, oito por recidiva e um por hemorragia. Morte antes dos 100 dias ocorreu em dois pacientes, um por recidiva e outro por hemorragia decorrente da plaquetopenia refratária, relacionada ao procedimento. Concluímos que o regime de condicionamento Bu/Mel é opção válida ao uso de outros regimes de condicionamento, apresentando excelente taxa da sobrevida. Rev. Bras. Hematol. Hemoter. 2008;30(5):352-358.
\end{abstract}

Palavras-chave: Transplante de células-tronco hematopoéticas; transplante autogênico; leucemia mielóide aguda; mortalidade; toxicidade de drogas.

\section{Introdução}

O transplante de células-tronco hematopoéticas (TCTH) de medula óssea (MO) ou de células progenitoras periféricas (CPP) tem sido utilizado no tratamento da leucemia mielóide aguda (LMA) em primeira remissão completa $\left(1^{\text {a }} \mathrm{RC}\right) \cdot{ }^{1-7}$ As opções na consolidação em pacientes de risco citogenético intermediário e alto têm sido o transplante alogênico, quando da disponibilidade de doador como decisão preferencial, autogênico em pacientes de risco intermediário ou a quimioterapia intensiva em pacientes de baixo risco citogenético. ${ }^{1-7}$ Vários grupos ${ }^{1-3}$ demonstraram redução do risco de recaída em adultos submetidos ao trans- plante da modalidade autogênica (TCTH Auto). Em seis estudos elegíveis por metanálise recente, conduzido por Nathan e cols, ${ }^{8}$ em que foi comparado o autotransplante em pacientes sem doador com antígeno de histocompatibilidade (HLA) idêntico, com quimioterapia não mieloablativa e observação após quimioterapia de indução, foi demonstrado que pacientes submetidos a autotransplante tiveram melhor sobrevida livre de doença, mas sobrevida global semelhante.

O TCTH Auto na LMA em $1^{\text {a }} \mathrm{RC}$ é realizado após quimioterapia de indução ${ }^{9-11} \mathrm{e}$ de um ou dois ciclos de consolidação intensiva, ${ }^{12,13}$ geralmente em pacientes com idade inferior a 60 anos, com prognóstico citogenético intermediá-

FMUSP - Disciplina de Hematologia e Hemoterapia - Serviço de TMO - HC-FMUSP - São Paulo-SP.

Correspondência: Nadjanara Dorna Bueno

Serviço de Transplante de Medula Óssea do Hospital das Clínicas da Faculdade de Medicina da USP

Avenida João Ramalho, 148 apto 32 - Centro

09030-320 - Santo André-SP - Brasil

Tel.: $55212506-6866$ / $55212506-6697$ / 552196078062

E-mail: nadjanaradb@ig.com.br 
rio, ${ }^{14-16} \mathrm{sem}$ fatores de risco para recaída, para os pacientes que não possuem doador compatível no sistema HLA. Como critério de estratificação destes pacientes utiliza-se a classificação Franco-Américo-Britânica (FAB), habitualmente se associando às alterações citogenéticas observadas. ${ }^{17-19}$

$\mathrm{O}$ condicionamento para o TCTH Auto nos diversos estudos é variado, sendo habitual a observação de regimes ora utilizando a irradiação corporal total associada à ciclofosfamida, ora a droga anterior descrita e o bussulfano, além de outros regimes menos freqüentes.

O uso do bussulfano e melfalano como regime de condicionamento no TCTH Auto leva em conta o grau destes agentes serem ativos no tratamento da LMA, porém pouco utilizados..$^{20-23}$ No nosso serviço, desde 1993, tem se utilizado o bussulfano e o melfalano como condicionamento em pacientes submetidos a autotransplante, com o racional de que a maioria destes pacientes previamente é mobilizada com ciclofosfamida (CFM) e fator de crescimento celular (GCSF) para utilização das CPP, e ainda com o objetivo de diminuir a incidência de hematúria relacionada ao uso da CFM.

$\mathrm{O}$ relato faz parte de projeto de estudo apresentado e aceito pelo Comitê de Análise de pesquisas da instituição, e todos os pacientes foram informados e consentiram de livre e espontânea vontade, em participar do estudo.

\section{Casuística e Métodos}

No período de 21 de dezembro de 1993 a 19 de janeiro de 2006, 22 pacientes consecutivos portadores de leucemia mielóide aguda (LMA) em primeira remissão completa $\left(1^{\text {a }} \mathrm{RC}\right)$ foram submetidos a transplante de células-tronco hematopoéticas autogênico (TCTH Auto), tendo a análise sido encerrada em 23 de janeiro de 2007.

A casuística está constituída de seis pacientes do sexo masculino e 16 do feminino. A mediana de idade na data do transplante foi de 26 anos, sendo que o mais jovem tinha 5 anos e o mais idoso, 58. Em relação à raça, 18 eram caucasóides e quatro não caucasóides. Um paciente apresentou o antecedente de tratamento quimioterápico por câncer de mama e outro tinha apresentado aplasia de medula óssea, dois anos antes do diagnóstico da LMA.

A análise citogenética foi efetuada em 12 pacientes, sendo considerado um de bom prognóstico, dez de prognóstico intermediário e um desfavorável. Em dez pacientes não havia dados do estudo citogenético. De acordo com a classificação FAB, dados revistos em nosso serviço, foram classificados dois pacientes do subtipo M0, oito como M1, sete M2, dois M4, e dois casos secundários por câncer de mama e aplasia de medula. Em um paciente não foi possível determinar o subtipo.

Doze pacientes foram induzidos com o protocolo quimioterápico composto por arabinosideo (Ara-C) durante sete dias e antraciclina por três dias (3+7), quatro pacientes com tioguanina, Ara-C e daunoblastina (TAD) e, em seis, foram utilizados outros protocolos de indução. Receberam quimioterapia de consolidação com altas doses de ara-C (ADAra-C) 15 pacientes, enquanto cinco não a receberam. Somente um paciente recebeu manutenção e em um não se obteve esta informação.

Após o $1^{\circ}$ ciclo de quimioterapia, oito pacientes obtiveram $\mathrm{RC}$, em sete ela não foi constatada. Em sete não havia esta informação quando do encaminhamento do paciente ao serviço.

\section{Mobilização}

Foram mobilizados com a ciclofosfamida $120 \mathrm{mg} /$ $\mathrm{kg} /$ dose subdividida em dois dias e adição de filgrastima (G-CSF) $10 \mu \mathrm{g} / \mathrm{kg} /$ dia desde o primeiro dia de granulócitos abaixo de 1,0X10 $/ 1$ até o término das sessões de aféreses de leucócitos para obtenção de células progenitoras periféricas (CPP). Nos casos em que não se obteve mobilização, foi utilizado, como fonte de células, a medula óssea (MO).

As células-tronco hematopoéticas infundidas tiveram como fonte a $\mathrm{MO}$ em seis pacientes, CPP em 13, MO e CPP em dois pacientes. Não havia informação sobre a fonte de células em um paciente. A mediana de células totais infundidas foi de $6,27 \times 10^{8} / \mathrm{kg}(1,3$ a 28,1$)$ e de células CD34+ foi $2,3 \times 10^{6} / \mathrm{kg}(0,24-3,37)$. Em MO, o número de células totais e

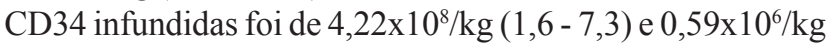
$\left(0,29\right.$ - 4,0) e em CPP foi respectivamente de $13,1 \times 10^{8} / \mathrm{kg}(1,3$ $-28,1)$ e $2,25 \times 10^{6} / \mathrm{kg}(0,24-3,37)$.

A mediana de transfusão de concentrado de hemáceas (CH) antes do transplante, em 12 pacientes avaliados, foi de oito unidades. ${ }^{1-22}$ Receberam plaquetas por aférese três pacientes, com uma mediana de quatro unidades. ${ }^{5-15}$ Plaquetas de múltiplos doadores (CPM) foram infundidas em sete pacientes, com mediana de 28 unidades.$^{8-177}$ Não foi possível determinar o número de transfusões prévias nos demais pacientes, por terem recebido múltiplas transfusões, ou por falta de informação ao encaminhamento.

\section{Regime de condicionamento}

Os pacientes foram condicionados com o bussulfano $16 \mathrm{mg} / \mathrm{kg}(1 \mathrm{mg} / \mathrm{kg}$ por via oral a cada seis horas em quatro dias consecutivos nos dias -7 a -4) e melfalano $140 \mathrm{mg} / \mathrm{m}^{2}$ no dia anterior ao transplante $(\mathrm{Bu} / \mathrm{Mel})$.

\section{Profilaxias}

Todos os pacientes receberam $10 \mathrm{mg} / \mathrm{kg} / \mathrm{dia}$ de difenilhidantoina, por via oral, dividida em quatro doses para prevenir crises convulsivas. Para prevenção de infecção receberam trimetropim-sulfametoxazol, cefepine e aciclovir até a pega do enxerto. As características dos pacientes submetidos ao transplante encontram-se na Tabela 1.

A análise estatística realizada considerou como evento de interesse o óbito. As estimativas de probabilidades de sobrevida acumulada foram feitas pelo estimado produto limite de Kaplan-Meier, e as comparações entre as curvas pelo 
teste de log-rank. Foi feita análise estatística descritiva do tempo de seguimento dos pacientes vivos em 23 de janeiro de 2007. Em todas as análises adotou-se o nível de significância $(p)$ de $5 \% .^{24}$

Os pacientes foram avaliados retrospectivamente, estavam em primeira remissão completa, e a indicação do TCTH foi decorrente da inexistência de doador HLA idêntico, para a realização do transplante alogênico. Foram analisadas a enxertia, a toxicidade relacionada ao regime de condicionamento, sendo os desfechos primários dos estudos a mortalidade relacionada ao tratamento (MRT), recaída de doença (Rec), e sobrevida global (SG) e livre de doença (SLD) dos pacientes.

Os critérios de classificação utilizados foram da classifícação $\mathrm{FAB},{ }^{17-19}$ de risco citogenético ${ }^{15,16}$ e de toxicidade da Organização Mundial de Saúde. ${ }^{25}$

\section{Definições}

Remissão completa (RC) é ausência de blastos no sangue periférico, menos de $5 \%$ de blastos na medula óssea, falta de evidências de leucemia extramedular ou ausência de sinais clínicos de recaída da doença.

Mortalidade relacionada ao transplante define-se com evento, o óbito nos primeiros 100 dias do transplante. Recaída como a recidiva da moléstia a qualquer momento após o transplante. SLD como sobrevida sem evidências de moléstia presente. Para análise de SLD, óbito ou recidiva são considerados como eventos para fins estatísticos. SG são os pacientes sobreviventes da casuística observados na data do último follow-up.

Enxertia granulocitária (pega) é a presença de mais $0,5 \times 10^{9} / 1$ de granulócitos em contagens hematológicas de três dias consecutivos. Enxertia plaquetária é a presença de mais de $20 \times 10^{9} / 1$ de plaquetas por mais de sete dias, sem a necessidade de transfusão plaquetária. O tempo de seguimento foi calculado como o intervalo entre a data do transplante até a data do óbito, ou até 23 de janeiro de 2007 , para os que estavam vivos quando se encerrou este estudo.

\section{Resultados}

Dos 22 pacientes avaliados, entre o diagnóstico informado e a data da realização do transplante, a mediana foi de 283 dias (164-1.129 dias). A mediana de seguimento dos pacientes foi de 635 dias (38-4.744). A mediana de enxertia foi de 14 dias em 21 pacientes avaliados para granulócitos superior a $0,5 \times 10^{9} / 1(0-38)$, para número de plaquetas em 20 pacientes, número superior a $20 \times 10^{9} / 1$ foi de 14 dias $(0-98) \mathrm{e}$

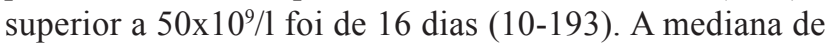
enxertia nos pacientes que receberam MO foi de 14 dias (018) para granulócitos, de 26 dias (14-98) para número de plaquetas superior a 20x10\%/, e de 62 dias (14-193) para número de plaquetas superior a $50 \times 10^{9} / 1$.
Tabela 1

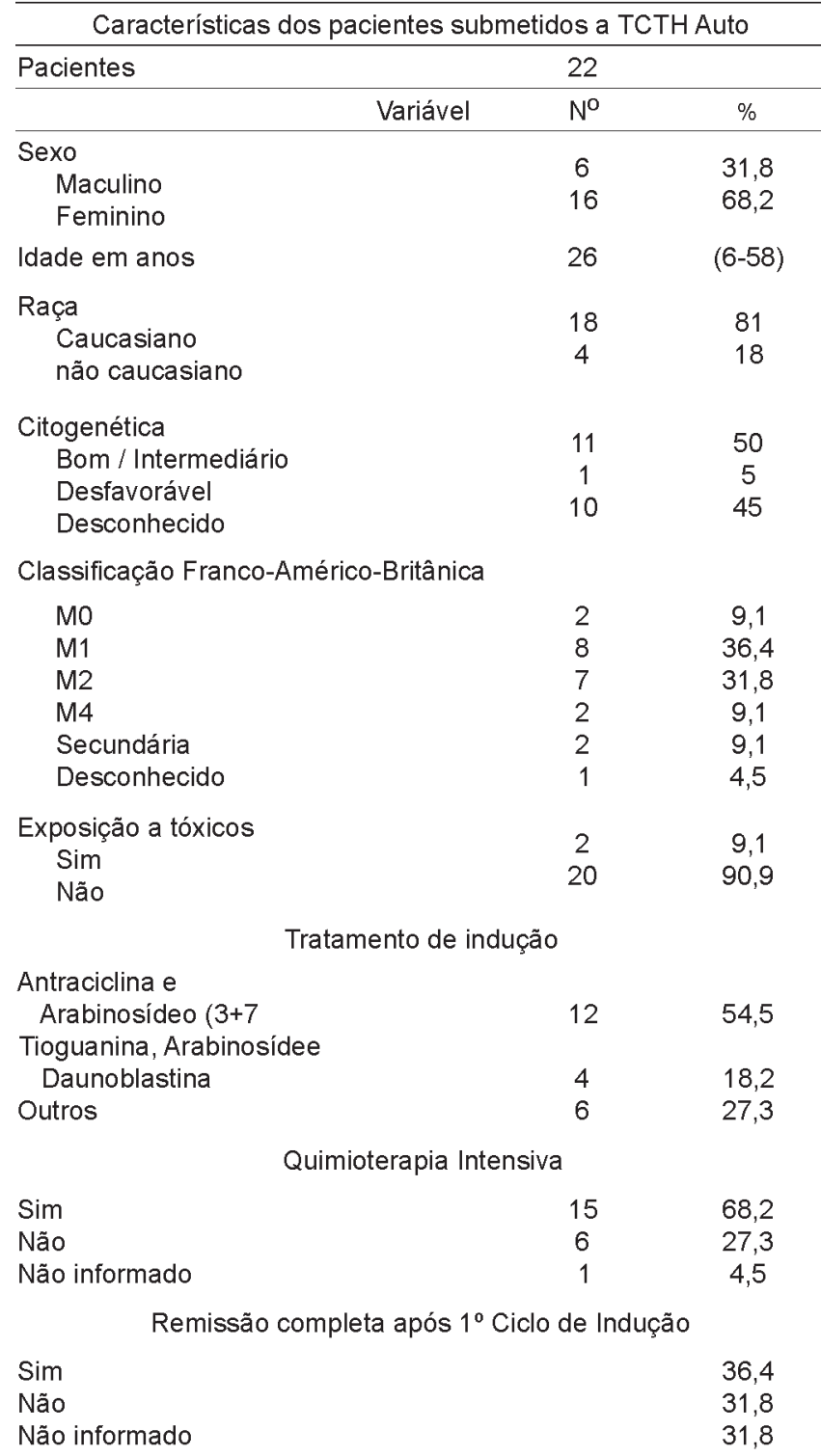

Fonte de Células

\begin{tabular}{lcc} 
Medula óssea & 6 & 27,2 \\
$\begin{array}{l}\text { Células progenitoras } \\
\text { periféricas }\end{array}$ & 13 & 59,1 \\
$\begin{array}{l}\text { Medula óssea + celulas } \\
\text { progenitoras periféricas }\end{array}$ & 2 & 9,1 \\
Não informado & 1 & 4,5 \\
\multicolumn{3}{c}{ Mediana de células infundidas } \\
Totais & $6,27 \times 10^{8} / \mathrm{kg}$ & $(1,3-28)$ \\
CD34+ & $2,3 \times 10^{6} / \mathrm{kg}$ & $(0,24-4,0)$ \\
\hline
\end{tabular}

Para os pacientes que receberam a CPP, a mediana de enxertia para granulócitos foi de 14 dias (10-38), para número de plaquetas superior a $20 \times 10^{9} / 1,10$ dias $(0-38)$, e para número de plaquetas superior a 50x10 $/ 1,16$ dias (10-51). Em um paciente houve recuperação medular com células blásticas, três semanas após a infusão das células. 
A mediana de transfusão após o TCTH para concentrado de hemáceas $(\mathrm{CH})$ filtradas e irradiadas em 22 pacientes foi de 5,5 unidades (1-16), a de concentrado de plaquetas (CPA) em 20 pacientes foi de 8,5 unidades (1-44) e de CPA em 11 pacientes foi de 21 unidades (4-216).

Doze pacientes apresentaram infecção, e em 10 não houve infecção constatada. A antigenemia para o CMV esteve positiva em cinco pacientes, do dia +2 ao +225 do TCTH, com mediana de positividade ao redor do dia +36 . A síndrome obstrutiva sinusoidal (SOS) não foi observada em nenhum paciente. Hematúria ocorreu em um paciente, provavelmente por plaquetopenia refratária.

A análise de enxertia e toxicidade dos pacientes submetidos ao procedimento encontra-se na Tabela 2 .

Tabela 2. Enxertia e toxicidade dos 22 pacientes submetidos a TCTH Auto condicionados co BU/Mel

\begin{tabular}{|c|c|c|c|}
\hline Mediana & Dias & $(\min -\max )$ & Total \\
\hline $\begin{array}{l}\text { Enxertia de neutrófilos } \\
>0,5 \times 10^{9} / / \text { (em dias) }\end{array}$ & 14 & $(0-38)$ & 21 \\
\hline $\begin{array}{l}\text { Plaquetas }>20 \times 10^{9} / 1 \\
\text { (em dias) }\end{array}$ & 15 & $(0-98)$ & 20 \\
\hline $\begin{array}{l}\text { Plaquetas }>50 \times 10^{9} / 1 \\
\text { (em dias) }\end{array}$ & 16 & $(10-193)$ & 20 \\
\hline Transfusão & Unidades & $(\min -\max )$ & Total \\
\hline Concentrado de hemácias & 5,5 & $(1-16)$ & 22 \\
\hline $\begin{array}{l}\text { Concentrado de plaquetas } \\
\text { por aférese }\end{array}$ & 8,5 & $(1-44)$ & 20 \\
\hline $\begin{array}{l}\text { Concentrado de plaquetas } \\
\text { de múltiplos doadores }\end{array}$ & 21 & $(4-216)$ & 11 \\
\hline $\begin{array}{l}\text { Sim Infecção } \\
\text { Não } \\
\text { Não aval }\end{array}$ & & $\begin{array}{c}12 \\
7 \\
3\end{array}$ & 22 \\
\hline Antigenemia (em dias) & 36 & $(21-225)$ & \\
\hline Para CVM $n=5$ & & $n^{0}$ & \\
\hline sos & $\begin{array}{l}\text { Não } \\
\text { Sim }\end{array}$ & $\begin{array}{c}22 \\
0\end{array}$ & \\
\hline Mucosite & $\begin{array}{c}1-2 \\
0 \\
3\end{array}$ & $\begin{array}{l}14 \\
4 \\
4\end{array}$ & \\
\hline Náuseas & $\begin{array}{c}1-2 \\
3 \\
0\end{array}$ & $\begin{array}{l}11 \\
2 \\
9\end{array}$ & \\
\hline Vômito & $\begin{array}{c}1-2 \\
3 \\
0\end{array}$ & $\begin{array}{l}9 \\
2 \\
11\end{array}$ & \\
\hline Diarréia & $\begin{array}{c}1-2 \\
3 \\
0\end{array}$ & $\begin{array}{l}9 \\
2 \\
11\end{array}$ & \\
\hline Hematúria & $\begin{array}{l}\text { Não } \\
\text { Sim }\end{array}$ & $\begin{array}{c}1 \\
21\end{array}$ & \\
\hline
\end{tabular}

min/máx - mínimo/máximo

N/Aval - Não avaliado

CVM - citomegalovírus

SOS - Síndrome Obstrutiva Sinusoidal
A taxa de SG nesta casuística foi de 57,5\% após 36 meses, com 13 pacientes vivos e sem doença ao final do estudo. A estatística descritiva dos pacientes que permaneceram vivos ao final mostrou uma mediana de sobrevida de 1.576 dias (369-4.744).

A probabilidade de sobrevida pelo método de KaplanMeier não mostrou diferença estatística significante em relação ao sexo, ao subtipo de leucemia pela classificação $\mathrm{FAB}$, ao protocolo de indução, à consolidação com quimioterapia de altas doses de arabinosídeo, remissão após o $1^{\circ}$ ciclo de indução ou a fonte de células infundidas (MO ou CPP). A análise citogenética teve significância estatística, pois o único paciente de prognóstico desfavorável morreu um ano após o transplante. Quando se compararam os pacientes de prognóstico bom/intermediário com os de prognóstico desconhecido, as probabilidades de sobrevida foram semelhantes.

$\mathrm{Na}$ tabela 3 apresentamos as probabilidades de sobrevida segundo as variáveis do estudo e sua significância estatística. As curvas de sobrevida global e livre de doença encontram-se nos gráficos 1 e 2.

Dos nove pacientes que foram a óbito, oito morreram por recidiva da leucemia e um por hemorragia. Foram a óbito antes dos cem dias, dois pacientes, um por recidiva no dia +38 pós-transplante e outro por hemorragia, que ocorreu por plaquetopenia refratária no dia +51 .

Dos pacientes que foram a óbito por recidiva, no diagnóstico da doença um apresentava hiperleucocitose superior a $100.00 / \mathrm{mm}^{3}$, e outro infiltração leucêmica em gânglios linfáticos. Pela classificação FAB, três eram do subtipo M1, dois M4, um M2, um M0 e um M7 secundária a aplasia de medula óssea, dois anos antes do procedimento. $\mathrm{O}$ estudo citogenético demonstrou dois pacientes com citogenética normal, um com hipodiploidia, uma análise citogenética complexa e, em quatro, era desconhecida. Tinham atingido remissão completa $(\mathrm{RC})$ após $1^{\circ}$ ciclo de indução quimioterápica seis pacientes, em um não havia sido atingido e em outro não havia informação quando do encaminhamento do paciente para o serviço de transplante. Foram submetidos a consolidação com $\mathrm{ADAra}-\mathrm{C}$ seis pacientes e dois não receberam ADAra-C.

\section{Discussão}

O tratamento da LMA atualmente se baseia na quimioterapia de indução com arabinosídeo e antraciclinas, seguida de consolidação intensiva com altas doses de arabinosídeo. A obtenção de remissão completa associada à alteração citogenética considerada de bom prognóstico posterga o uso de terapias mais agressivas como o transplante para uma segunda linha, ou após recidiva. A obtenção de RC nos pacientes considerados de prognóstico intermediário ou desfavorável tem como indicação na existência de doador HLA idêntico, a consolidação com o TCTH alogênico. A 
Tabela 3. Probabiliade de sobrevida dos pacientes condcionados com Bu/Mel no TCTH Auto em $1^{\text {a }}$ remissão completa

\begin{tabular}{cccccc}
\hline \multirow{2}{*}{ Variável } & Categoria & \multicolumn{3}{c}{ Taxa de sobrevida } & \multirow{2}{*}{ (em meses) } \\
& & $12 \mathrm{~m}$ & $24 \mathrm{~m}$ & $36 \mathrm{~m}$ & \\
\hline \multirow{2}{*}{ Sexo } & Masculino & 100 & 66,7 & 66,7 & 0,5567 \\
Tipo de Leucemia & Feminino & 68,7 & 55 & 55 & \\
Mielóide Aguda (FAB) & M1 + M2 + M4 & 70,6 & 55,8 & 55,8 & 0,9337 \\
& Outros & 100 & 50 & 50 & \\
Análise & BP/PI & 72,3 & 62,3 & 62,3 & \multirow{2}{*}{0,0066} \\
citogenética & P Desf & 0 & 0 & 0 & \\
& P Desc & 90 & 60 & 60 & \\
Tratamento de Indução & 3+7/ TAD & 68,8 & 48,1 & 48,1 & 0,1475 \\
& Outros & 83,3 & 83,3 & 83,3 & \\
Consolidação & Sim & 68,7 & 55 & 55 & 0,3256 \\
Intensiva & Não & 100 & 80 & 80 & \\
Remissão após & Sim & 62,5 & 25 & 25 & \multirow{2}{*}{0,0816} \\
$1^{\circ}$ ciclo & Não & 100 & 80 & 80 & 0,0816 \\
& N/Inf & 75 & 75 & 75 & \\
Fonte de células & MO/MO/CPP & 77,8 & 66,7 & 66,7 & 0,7331 \\
& CPP & 75 & 54,7 & 54,7 & \\
\hline & Total & 77,3 & 57,5 & 57,5 & \\
\hline
\end{tabular}

BP/PI - Bom Prognóstico / Prognóstico Intermediário; P Desf. - Prognóstico Desfavorável;

$P$ Desc. - Prognóstico Desconhecido;

3+7/TAD - Antraciclina + Arabinosídeo / Tioguanina, Antraciclina e Daunoblastin;

$\mathrm{N} /$ /nf. - Não Informado; MO/MO+CPP - Medula óssea / medula óssea e células progenitoras periféricas; $p^{*}$ - significância estatística

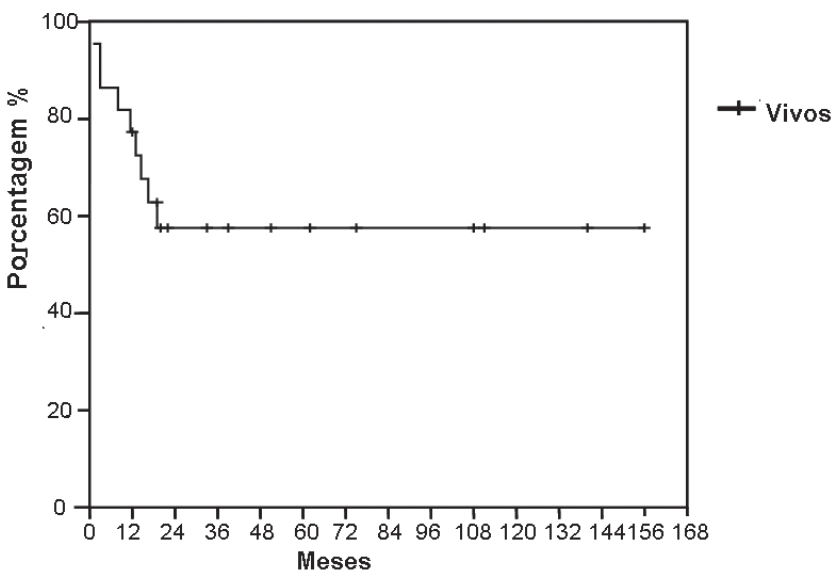

Gráfico 1. Sobrevida global dos pacientes submetidos a transplante autogênico na LMA em $1^{\text {a }} \mathrm{RC}$, condicionados com Bu/Mel

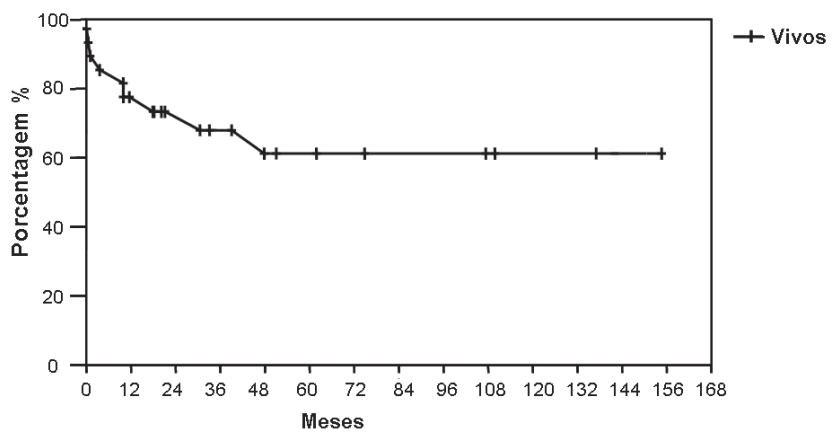

Gráfico 2. Sobrevida livre de doença dos pacientes submetidos a transplante autogênico na LMA em $1^{\circ} \mathrm{Rc}$, condiconados com Bu/Mel inexistência de doador coloca os pacientes para a opção do TCTH Auto ou quimioterapia de consolidação.

O TCTH Auto ainda precisa de dados mais conclusivos para ter a sua indicação efetivada, mas vários relatos o colocam como uma opção válida principalmente após a primeira remissão completa, quando da indisponibilidade de um doador, que foi o escopo deste relato.

Os regimes de condicionamento mais comumente utilizados são os que associam a irradiação corporal total ou a ciclofosfamida ao bussulfano. Os outros programas são mais raros e a associação de Bu/Mel mais rara ainda. Esta associação visa reduzir a toxicidade vesical, que é observada quando do uso de ciclofosfamida. ${ }^{26,27}$ Outro dado a ser observado e que pode justificar esta associação são os pacientes que apresentam baixa fração de ejeção ventricular $(<55 \%)$, em que a toxicidade da ciclosfofamida deve ser evitada, além da cistite hemorrágica associada ao uso da mesma. Outro fator ao uso do melfalano refere-se à sua baixa toxicidade e, principalmente, ao fato que não agrega toxicidade na associação com o bussulfano, e ao fato de a ciclofosfamida ser utilizada na mobilização da CPP.

No presente estudo com $\mathrm{Bu} / \mathrm{Mel}$ como regime de condicionamento para o TCTH Auto, os resultados demonstram a efetividade deste regime de condicionamento, com baixa toxicidade em relação a mucosite, enterite, pneumonia intersticial, SOS e hematúria. Bussulfano e ciclofosfamida $(\mathrm{Bu} / \mathrm{CFM})^{27}$ e irradiação corporal total e ciclofosfamida (ICT/ $\mathrm{CFM})^{26}$ são particularmente efetivos como condicionamento, porém levam a alta toxicidade. ${ }^{28}$ Mucosite leve e moderada ocorreu com alguma freqüência, mas nenhum paciente apresentou SOS e somente um paciente apresentou hematúria, sendo esta relacionada à plaquetopenia refratária, e não a cistite hemorrágica.

Doze pacientes apresentaram infecção pós-transplante e a antigenemia para CMV esteve positiva em somente cinco pacientes. A mediana de enxertia não diferiu dos dados obtidos da literatura. ${ }^{29,30}$

Com este regime de condicionamento, a probabilidade de sobrevida global se situou em torno de 57,5\% aos 36 meses após o transplante, com 13 dos 22 pacientes, vivos e sem doença, similares ao observado na literatura..$^{31,32}$

Observamos um plateau na curva de sobrevida pelo método de Kaplan-Meier aos 20 meses após-transplante, dado este já demonstrado em outro relato ${ }^{33}$ em que ocorreu o plateau aos 24 meses. 
Dos nove pacientes que foram a óbito, oito ocorreram por recidiva e o outro foi por toxicidade (plaquetopenia refratária) no dia 51 após o transplante. Nenhum paciente foi a óbito em decorrência de infecção.

Concluímos que o TCTH Auto em $1^{\mathrm{a}} \mathrm{RC}$ na LMA em pacientes que receberam como regime de condicionamento $\mathrm{Bu} / \mathrm{Mel}$ apresentou baixa mortalidade relacionada ao procedimento, sendo opção válida ao uso de $\mathrm{Bu} / \mathrm{CFM},{ }^{26,27}$ por diminuir a incidência de cistite hemorrágica, apresentando excelente taxa de sobrevida.

\begin{abstract}
Twenty-two consecutive patients with acute myeloid leukemia in first complete remission submitted to autologous hematopoietic stem cells transplantation conditioned with busulfan and melphalan were evaluated between 1993 and 2006. The overall survival, according to the Kaplan-Meier curve, was $57.5 \%$ at 36 months, with a "plateau" at 20 months after transplant. Factors such as gender, FrenchAmerican-British (FAB) classification of acute myeloid leukemia, induction therapy, intensive consolidation, remission after the first cycle of induction and source of cells had no impact on survival. One patient with poor prognosis before the procedure died a year after transplantation. Nine patients died, eight by relapse and one because of bleeding. Death before 100 days occurred for two patients, one due to relapse and the other bleeding caused by refractory thrombocytopenia related to the procedure. In conclusion, the conditioning regiment with busulfan and melphalan is a valid option compared to the other conditioning regimens, with an excellent overall survival. Rev. Bras. Hematol. Hemoter. 2008;30(5):352-358.
\end{abstract}

Key words: Hematopoietic stem cell transplantation; autologous transplantation; acute myeloid leukemia; mortality; toxicity of drugs.

\section{Referências Bibliográficas}

1. Harousseau JL, Cahn JY, Pignon B et al. Comparison of autologous bone marrow transplantation and intensive chemotherapy as postremission therapy in adult acute myeloid leukemia. Blood. 1997;90(8):2978-86.

2. Zittoun RA, Mandelli F, Willemze R et al. AAutologous or allogeneic bone marrow transplantation compared with intensive chemotherapy in acute myelogenous leukemia. N Engl J Med. 1995;332(4):217-23.

3. Burnett AK, Goldstone AH, Stevens RM et al. Randomised comparison of addition of autologous bone-marrow transplantation to intensive chemotherapy for acute myeloid leukaemia in first remission: results of MRC AML 10 trial. Lancet. 1998; 351 (9104): 700-8.

4. Suciu S, Mandelli F, de Witte T, et al. Allogeneic compared with autologous stem cell transplantation in the treatment of patients younger than 46 years with acute myeloid leukemia (AML) in first complete remission (CR1): an intention-to-treat analysis of the EORTC/GIMEMAAML-10 trial. Blood. 2003;102(4):1232-40.

5. Cornelissen JJ, van Putten WL, Verdonck LF et al. Results of a HOVON/SAKK donor versus no-donor analysis of myeloablative HLA-identical sibling stem cell transplantation in first remission acute myeloid leukemia in young and middle-aged adults: benefits for whom? Blood. 2007;109(9):3658-66.
6. Cassileth PA, Harrington DP, Appelbaum FR et al. Chemotherapy compared with autologous or allogeneic bone marrow transplantation in the management of acute myeloid leukemia in first remission. N Engl J Med. 1998;339(23):1649-56.

7. Keating S, de Witte T, Suciu S et al. The influence of HLAmatched sibling donor availability on treatment outcome for patients with AML: an analysis of the AML 8A study of the EORTC Leukaemia Cooperative Group and GIMEMA. Br J Haematol. 1998;102(5):1344-53.

8. Nathan PC, Sung L, Crump M, Beyene J. Consolidation therapy with autologous bone marrow transplantation in adults with acute myeloid leukemia: a meta-analysis. J Natl Cancer Inst. 2004; 96 (1):38-45.

9. Rai KR, Holland JF, Glidewell OJ et al. Treatment of acute myelocytic leukemia: a study by cancer and leukemia group B. Blood. 1981;58(6):1203-12.

10. Berman E, Heller G, Santorsa J et al. Results of a randomized trial comparing idarubicin and cytosine arabinoside with daunorubicin and cytosine arabinoside in adult patients with newly diagnosed acute myelogenous leukemia. Blood. 1991;77(8):1666-74..

11. Arlin Z, Case DC Jr, Moore J, Cartwright K et al. Randomized multicenter trial of cytosine arabinoside with mitoxantrone or daunorubicin in previously untreated adult patients with acute nonlymphocytic leukemia (ANLL). Leukemia. 1990; 4 (3):177-83.

12. Bishop JF, Matthews JP, Young GA et al. A randomized study of high-dose cytarabine in induction in acute myeloid leukemia. Blood. 1996;87(5):1710-7.

13. Mayer RJ, Davis RB, Schiffer CA et al. Intensive postremission chemotherapy in adults with acute myeloid leukemia. Cancer and Leukemia Group B. N Engl J Med. 1994;331(14):896-903.

14. Byrd JC, Mrózek K, Dodge RK et al. Pretreatment cytogenetic abnormalities are predictive of induction success, cumulative incidence of relapse, and overall survival in adult patients with de novo acute myeloid leukemia: results from Cancer and Leukemia Group B (CALGB 8461). Blood. 2002;100(13):4325-36.

15. Mrózek K, Heinonen K, de la Chapelle A. et al. Clinical significance of cytogenetics in acute myeloid leukemia. Semin Oncol. 1997; 24(1):17-31.

16. Grimwade D, Walker H, Oliver F et al. The importance of diagnostic cytogenetics on outcome in AML: analysis of 1,612 patients entered into the MRC AML 10 trial. The Medical Research Council Adult and Children's Leukaemia Working Parties. Blood. 1998;92(7):2322-33.

17. Bennett JM, Catovsky D, Daniel MT et al. Proposals for the classification of the acute leukaemias. Br J Haematol. 1976; 33 (4):451-8.

18. Bennett JM, Catovsky D, Daniel MT et al. Proposed revised criteria for the classification of acute myeloid leukemia. A report of the French-American-British Cooperative Group. Ann Intern Med. 1985;103(4):620-5.

19. Bennett JM, Catovsky D, Daniel MT et al. Criteria for the diagnosis of acute leukemia of megakaryocyte lineage (M7). A report of the French-American-British Cooperative Group. Ann Intern Med. 1985;103(3):460-2.

20. Martino R, Badell I, Brunet S et al. High-dose busulfan and melphalan before bone marrow transplantation for acute nonlymphoblastic leukemia.. Bone Marrow Transplant. 1995; 16(2):209-12.

21. Vignetti M, Rondelli R, Locatelli F et al. Autologous bone marrow transplantation in children with acute myeloblastic leukemia: report from the Italian National Pediatric Registry (AIEOP-BMT). Bone Marrow Transplant. 1996;18 Suppl 2:59-62. 
22. Marit G., Cony P, David B. High-dose busulfan and melphalan as conditioning regiment prior to transplantation. Bone Marrow Transplant. 1988;3:78, Suppl 1.

23. Imrie K, Dicke KA, Keating A. Autologous bone marrow transplantation for acute myeloid leukemia. Stem Cells. 1996;14 (1):69-78.

24. Lee ET, Wang JW. Statistical methods for survival date analysis, 3rd. New Jersey. 2003. John Wiley \& Sons Inc.

25. Miller $\mathrm{AB}$, Hoogstraten $\mathrm{B}$, Staquet $\mathrm{M}$ et al. Reporting results of cancer treatment. Cancer. 1981;47(1):207-14.

26. Thomas ED, Clift RA, Hersman J et al. Marrow transplantation for acute nonlymphoblastic leukemic in first remission using fractionated or single-dose irradiation. Int J Radiat Oncol Biol Phys. 1982;8(5):817-21.

27. Tutschka PJ, Copelan EA, Klein JP. Bone marrow transplantation for leukemia following a new busulfan and cyclophosphamide regimen. Blood. 1987;70(5):1382-8.

28. Kernan NA, Bartsch G, Ash RC et al. Analysis of 462 transplantations from unrelated donors facilitated by the National Marrow Donor Program. N Engl J Med. 1993;328(9):593-602.

29. Storb R, Prentice RL, Thomas ED. Marrow transplantation for treatment of aplastic anemia. An analysis of factors associated with graft rejection. N Engl J Med. 1977;296(2):61-6.

30. Hill RS, Mazza P, Amos D et al. Engraftment in 86 with patients lymphoid malignancy after autologous marrow transplantationn. Bone Marrow Transplant. 1989;4(1):69-74.

31. O'Donnell MR. The role of autologous and allogeneic (full and mini) stem cell transplantation in AML. Annual Meeting, American Society of Hematology. 2004; San Diego, 104-9.

32. The CIBMTR: Current use and outcome of hematopoietic stem cell transplantation. [CD-Rom]; 2005.

33. Ganguly S, Singh J, Divine CL et al. Is there a plateau in the survival curve after autologous transplantation in patients with intermediate and high-risk acute myeloid leukemia? A 20-year single institution experience. Leuk Res. 2007;31(9):1253-7.

Avaliação: Editor e dois revisores externos

Conflito de interesse: não declarado

Recebido: 16/12/2007

Aceito após modificações: 12/03/2008 